\title{
Assessment of the Dense Granule Storage Pool in Migraine Patients
}

\author{
William T. Gunning* and Indrek Meyer** \\ *Departments of Biochemistry and Cancer Biology; and Pathology, College of Medicine, \\ Medical University of Ohio, Toledo, $\mathrm{OH} 43614$ \\ **College of Medicine, Medical University of Ohio, Toledo, $\mathrm{OH} 43614$.
}

Most patients evaluated in our laboratory for platelet (PL) dense granule (DG) storage pool deficiency $(\delta$-SPD) usually present clinically with significant bleeding histories including frequent epistaxis, easy bruising, heavy menses, excessive post surgical bleeding, and/or a family history of bleeding. We have often noted in the clinical histories provided with blood samples that a number of these patients suffer migraine headaches. This observation has led to a pilot study to evaluate the DG storage pool in migraine patients. A significant body of literature exists that either implicates or negates the role of the platelet in the etiology of migraine headaches. Some reports have suggested migraine is a "low serotonin syndrome". Migraine predominantly affects women of childbearing age, and is often associated with the menstrual period. We have previously reported a very high prevalence of decreased platelet (PL) dense granule (DG) number and volume in women clinically diagnosed with menorrhagia. The platelet DG contents include adenine nucleotides, calcium, and serotonin. The majority of serotonin in the blood is found stored in the platelet. If migraine is truly related to a low serotonin state, it would be reasonable to postulate that decreased numbers of PL DGs could be related to migraine headache, since a decrease in DG number would produce a decrease in storage pool constituents including serotonin. In this study, we have evaluated the PL DG number and adenine nucleotide content in female migraineurs $(n=40)$ between the ages of 20-50 (mean=37.6). The mean DG number (2.48 \pm 0.32 DG/PL) and elevated ATP/ADP ratio (5.49) is consistent with DG storage pool deficiency. Control samples obtained from females of similar age $(n=33)$ had $4.42 \pm 0.21$ DG/PL and an average ATP/ADP ratio of 1.72 which is consistent with normal values published in the literature and also in our lab (normal values: 4-6 DG/PL; ATP/ADP ratio: 1.4-1.9). The preliminary results of this study suggest that migraineurs are often platelet $\delta$-SPD. We would propose that a decrease in serotonin in platelet dense granules and/or in analogous granules in neural or other tissues may contribute to the pathogenesis of migraine headache. 